\title{
The effect of vacancies on the microwave surface resistance of niobium revealed by positron annihilation spectroscopy
}

\author{
A. Romanenko, ${ }^{1, a)}$ C. J. Edwardson, ${ }^{2}$ P. G. Coleman, ${ }^{2}$ and P. J. Simpson ${ }^{3}$ \\ 1) Fermi National Accelerator Laboratory, Batavia, IL 60510, USA \\ ${ }^{2)}$ Department of Physics, University of Bath, Bath BA2 7AY, UK \\ ${ }^{3)}$ Department of Physics and Astronomy, The University of Western Ontario, London, Ontario, \\ Canada N6A $3 K^{\gamma}$
}

(Dated: 25 May 2013)

Using variable-energy positron annihilation spectroscopy, we demonstrate that a different near-surface vacancy concentration accompanies drastic differences in surface resistance of superconducting niobium cavities for particle acceleration. Our data suggests that vacuum baking at $120^{\circ} \mathrm{C}$ leads to the doping of a near-surface layer with vacancy-hydrogen complexes, and that higher vacancy-type defect concentration distinguishes electropolished from chemically etched cavities. Our findings may help to explain a strong dependence of cavity performance on heat and chemical treatments, and may be of interest to other physics fields including cavity QED, microresonators, and single photon detectors.

The microwave surface impedance of superconductors $\$ 12$ describes the absorption and reflection of electromagnetic waves at the surface. Among conventional superconductors, the surface impedance of niobium has received much attention lately due to its importance for superconducting cavities for particle acceleration $3 \sqrt{4}$, microresonators 5 , cavity $\mathrm{QED}^{6}$, and single-photon detectors ${ }^{7}$. Although operated in different temperature and field regimes, the common unresolved problem for the applications above is the nature of defects within the magnetic penetration depth $\lambda \sim 40 \mathrm{~nm}$ and their effect on surface impedance. Such defects, including vacancies, dislocations, and interstitial and substitutional impurities are unavoidable but their type and concentration may be altered by different heat and chemical treatments. Therefore, for effective niobium surface engineering and to elucidate the underlying physics it is of immense value to gain an understanding of the processes involved in various treatments, especially so for the ones with a strong effect on surface impedance.

Of particular importance for superconducting radio frequency $(\mathrm{SRF})$ cavities for particle acceleration is the real part of the impedance - surface resistance $R_{\mathrm{S}}$, which determines the required cryogenic power and limits achievable accelerating gradients. Surface treatments on SRF niobium cavities developed over the past decades provide excellent reproducibility ${ }^{4 / 8}$ and their effect on the average $R_{\mathrm{S}}$ has been firmly established. Yet the underlying physics is not clear, which is the subject of our work.

State-of-the-art treatments to sustain the highest surface magnetic fields in SRF cavities include electropolishing (EP), buffered chemical polishing (BCP), and a so called "mild baking" (MB). Mild baking is annealing of a cavity in vacuum at $90-120^{\circ} \mathrm{C}$ for 48 hours, which, when applied to electropolished or chemically etched niobium, drastically changes both the low field value of

\footnotetext{
a) Electronic mail: aroman@fnal.gov
}

the microwave surface resistance $R_{\mathrm{s}}$ and its field dependence ${ }^{9}$ 13. A large number of structural investigations were performed to gain better understanding of the effect $^{14}$, yet the underlying mechanisms governing the changes are still elusive. Such previously investigated mechanisms include surface roughness 15 , oxygen diffusion 16 17, and magnetic impurities in the oxide ${ }^{18}$. However, experimental evidence for these mechanisms is contradictory hinting that they may only play a secondary role. The latest experiments 1921 and theoretical proposals 22 suggest that dislocations, vacancies, and vacancyhydrogen complexes may be the primary defects whose presence and evolution is behind the variations in the $R_{\mathrm{s}}$ caused by different treatments.

In this Letter we present direct evidence of the effect of near-surface vacancy-type defects on the superconducting surface resistance of niobium, obtained by variable energy positron annihilation spectroscopy (VEPAS) on samples with measured $R_{\mathrm{S}}(B)$ in the superconducting state. Using rf characterized samples directly cut from cavities distinguishes our findings from previous investigations on niobium and allows us to avoid ambiguities introduced by otherwise unavoidable differences in the processing history between samples and cavities. By performing subsequent treatments on such samples we compare the corresponding surface resistance changes with the evolution of the defects.

In order to directly connect the defect structure to the rf performance, the best and most straightforward way is to perform temperature mapping measurements on state-of-the-art superconducting RF cavities followed by dissection of areas of interest from the walls and subsequent nanostructural investigations on the extracted samples. Such an approach has been proven to be extremely useful by past investigations 2023 , and we follow the same methodology here. Areas of extraction for subsequent VEPAS studies were identified by temperature mapping of cavity walls during rf measurements. Some samples underwent further treatments (BCP, MB) after extraction to replicate cavity processing before VEPAS 
data were obtained. Parameters of the cavities and corresponding samples are listed in Table I. Below we summarize details of the rf and temperature mapping measurements.

We used three different elliptical cavities made of high purity niobium for our studies: two fine grain $(\sim 50 \mu \mathrm{m})$ cavities of TESLA shape ${ }^{24}$ with the fundamental $T M_{010}$ mode at the resonant frequency $f_{0}=1.3 \mathrm{GHz}$, and one large grain $(\sim 10 \mathrm{~cm})$ cavity of Mark-III shape with $f_{0}=1.5 \mathrm{GHz}$. Fine grain cavities were electropolished for $\geq 120 \mu \mathrm{m}$ material removal, and one of the cavities was additionally baked under vacuum at $120^{\circ} \mathrm{C}$ for 48 hours. The large grain cavity was chemically polished (BCP) instead of electropolishing. Before rf measurements all cavities were high pressure water rinsed, which is a standard treatment to avoid field emission caused by microparticles. Using standard phase-lock techniques ${ }^{25}$ the intrinsic quality factor $Q_{0} \propto 1 / \bar{R}_{\mathrm{s}}$ $\left(\bar{R}_{\mathrm{S}}=\frac{\omega \mu_{0} \int_{V} H^{2} d V}{\int_{\mathrm{A}} R_{\mathrm{s}}(H) H^{2} d A}\right)$ was measured for each of the cavities at $2 \mathrm{~K}$ as a function of the peak surface magnetic field $B_{\text {peak }}$. Fig. 1 1a shows corresponding excitation curves $Q_{0}\left(B_{\text {peak }}\right)$ for both fine grain cavities. The $Q_{0}\left(B_{\text {peak }}\right)$ curve for the large grain cavity was similar to the unbaked fine grain cavity.

A custom-built temperature mapping system similar to that described in ${ }^{26}$ was attached to the outside of the cavity walls during the measurements. Spatial maps of the temperature difference $\Delta T=T_{\text {on }}-T_{\text {off }}$ with and without rf power in the cavity were obtained at different rf field levels. Such $\Delta T$ is proportional to the local power dissipation $P_{\mathrm{c}} \propto R_{\mathrm{s}}(H) H^{2}$, where $R_{\mathrm{s}}(H)$ is the local surface resistance, and $H$ is the local magnetic field. In Fig. 1f the magnitude of the local magnetic field at each sensor location and characteristic temperature maps are shown for baked and unbaked fine grain cavities at the same $B_{\text {peak }}=119 \mathrm{mT}$. Individual $\Delta T(B)$ for samples 1-EP and 3-EP-MB circled on the maps are shown in Fig. 1b. Samples 2-EP and 6-BCP had $\Delta T(B)$ curves of the shape similar to 1-EP, and that for 4-BCP would be expected to be the same. All mild baked samples (1-EP-MB, 2-EP-MB, 5-BCP-MB, and 7-BCP-MB) are characterized by the rf behavior similar to 3-EP-MB as known from previous studies.

In VEPAS positrons are implanted into a sample with energies $E$ between 0.25 and $30 \mathrm{keV}$. These energies dictate the positron implantation profile enabling depth profiling from the surface to a depth dependent principally on the materials density. Implanted positrons rapidly thermalize and diffuse to either annihilate from the freely diffusing state or become trapped in vacancy-type defects and interfaces, finally annihilating with the emission of two approximately anti-collinear $511 \mathrm{keV} \gamma$-rays. The momentum of the electrons at the annihilation site causes the $511 \mathrm{keV}$ line to broaden; this broadening is measured using a high-purity Ge detector and characterized using the $S$ and $W$ parameters defined as the fraction of the annihilation line in the central and outer regions, re- spectively. The $S$ parameter has a characteristic value for each type of annihilation site including vacancy-type defects. Vacancy-type defects result in an elevated $S$ parameter due to reduced overlap of the positron wavefunction with high momentum core electrons. Analysis of $S$ as a function of incident positron energy, $S(E)$, can provide depth-dependent information. Plots of $S$ versus $W$ can be used to examine individual annihilation states within the material27.

$S(E)$ plots for BCP samples (6-BCP, 7-BCP-MB, 4BCP, 5-BCP-MB) are shown in Fig. 2 and the nearsurface response in the inset. In what follows the $S$ parameter values are normalized to the bulk value in 7 BCP-MB. The mean depth $\left(z_{\mathrm{m}}\right)$ scale is obtained using the approximate expression $z_{\mathrm{m}}=4.7 E^{1.6} \mathrm{~nm}$, with $E$ in $\mathrm{keV}$. The data indicate an increase in the $S$ parameter for positron energies $E \leq 4 \mathrm{keV}$ as the only result of a $120^{\circ} \mathrm{C}$ bake. Corresponding $S$-W plots in Fig. $2 \mathrm{p}$ provide evidence that the change in response at low $E$ is not measurably different from that to defects deeper inside the sample, since the $S, W$ points lie, to within uncertainties, on the same line joining defected to undefected niobium states. Various positron 'states' such as different defect species result in characteristic annihilation lineshapes, and therefore characteristic $S$ and $W$ values. Points lying along a straight line in an $S$-W plot are typically representative of a single defect species with varying concentration.

$S(E)$ profiles for EP samples (1-EP, 2-EP, 3-EP-MB) are shown in Fig. 3 and the near-surface response in the inset. Higher $S$ parameters than in BCP samples are observed before mild baking throughout all the positron energies $E \leq 30 \mathrm{keV}$, corresponding to a depth of up to about $1 \mu \mathrm{m}$. Two extra peaks in $S$ at about 8.5 and $15 \mathrm{keV}$ distinguish the responses of 1-EP and 2-EP. An $S-W$ plot for these data, not shown, shows that all these high $S$ points lie on the same line shown in Fig. $2 \mathrm{~b}$, extrapolated beyond its top left corner. Baking results in a decrease of $S(E)$ for all energies, including the removal of the extra peaks, and $S$ - $W$ moves along the line towards the bulk $\mathrm{Nb}$ response at $(1,1)$ although not approaching it as closely as for the BCP samples in Fig. $2 \mathrm{p}$.

For interpretation of our results it is important to consider the vacancy-hydrogen interaction. It has been discovered $^{288}$ that, in the presence of hydrogen, the vacancy formation energy in many metals is strongly lowered. The phenomenon was named "superabundant vacancies" $(\mathrm{SAV})^{29130}$ and has been found in many M-H systems. V$\mathrm{H}$ complexes were also observed in large concentrations in H-loaded niobium ${ }^{3132}$. Theoretical calculations show ${ }^{33}$ that the number $n$ of hydrogen atoms trapped at each of the vacancies in niobium may range from one to six and the $S$ parameter response in Doppler broadening PAS of $\mathrm{V}-n \mathrm{H}$ complexes is a function of $n$. Higher $n$ corresponds to lower $S$ due to the lower effective open volume available to positrons. Furthermore, V-H complexes can be introduced into $\mathrm{Nb}$ by diffusion from the surface at elevated temperatures ${ }^{34}$. Such diffusion was found to hap- 
pen via "fast" and "slow" processes, believed to be due to diffusion along dislocations and to bulk diffusion.

With regard to the present studies, near-surface hydrogen segregation has been experimentally observed $\frac{23135}{2}$ on similar samples. Hence, V-H formation should be energetically favorable if kinetics allow. It is therefore plausible that the $S$ parameter increase at $E \leq 4 \mathrm{keV}$ after baking in the BCP samples 5-BCP-MB and 7-BCP-MB, as compared to the unbaked 4-BCP and 6-BCP in Fig. 2, can be attributed to $\mathrm{V}-\mathrm{H}$ complexes introduced by diffusion from the surface - let us call it process I.

In the electropolished samples the situation appears to be more complicated. Higher $S(E)$ in 1-EP and 2EP (Fig. 3) compared to BCP samples in Fig. 2 may be attributed to open-volume defects introduced by the EP treatment itself. As a result of baking, the $S$ parameter at all $E$ up to $30 \mathrm{keV}$ is decreased (1-EP-MB, 2-EP-MB, 3 -EP-MB in Fig. 3p and, furthermore, a change in the shape of $S(E)$ at the lower energies is observed (see inset, Fig. 3) similar to BCP samples. Under the assumption that EP-introduced defects are also of the $\mathrm{V}-n \mathrm{H}$ type, the extended $S(E)$ decrease may be caused by the increase in $\mathrm{H}$ occupancy $n$, which, as discussed above, leads to the decrease in $S$. Then a characteristic upward change in the shape of $S(E)$ at lower energies $E$ (see inset, Fig. 3) may be possibly explained by the simultaneously present inward V-H surface diffusion process, consistent with observations on BCP samples.

Several conclusions can be made from our studies with regard to SRF cavities.

Most importantly, the superconducting surface resistance of the investigated samples strongly correlates with the shape of the $S(E)$ curve. More specifically, the presence of a near-surface layer rich in V-type defects of a particular kind, arguably V-H complexes, leads to the absence of a strong rf dissipation at high magnetic fields. Doping of a thin surface layer of $\sim 50 \mathrm{~nm}$ with stable $\mathrm{V}-\mathrm{H}$ complexes may therefore be a major mechanism behind mild baking.

Our observations fit within the recently proposed ${ }^{\sqrt{22}}$ hypothesis of nanohydrides, which states that interstitial hydrogen segregated near the surface can form small niobium hydrides upon cooldown to typical cavity operating temperatures below $4.4 \mathrm{~K}$. Such hydrides are only superconducting by proximity effect and may cause the high field degradation of surface resistance. In ${ }^{22} \mathrm{~V}-\mathrm{H}$ complexes are considered as possible nucleation centers for hydrides when there is high concentration of unbound hydrogen available. Here we consider another possibility that the number of $\mathrm{V}-\mathrm{H}$ complexes is high enough to trap most of hydrogen. Similarly to impurity trapping that has been shown to prevent the formation of hydrides in cavities made out of dirty, low residual resistivity ratio (RRR) niobium $\frac{36}{36}$, vacancies introduced by baking may trap near-surface interstitial hydrogen and suppress niobium hydride formation. Such trapping will lead to a change in primary electron scattering centers at low temperatures (scattered $\mathrm{H}$ vs. $\mathrm{NbH}_{x}$ lumps) lead- ing to a lower electron mean free path after mild baking found from BCS theory modeling of low field surface resistance $\frac{10}{10}$ and, more recently, by direct $\mu \mathrm{SR}$ investigations of Meissner screening 8 .

Finally, our findings may help in understanding the superiority of EP over BCP in fine grain cavities ${ }^{4}$. Both higher onset fields of strong dissipation before baking and an improved mild baking efficiency may be related to the pre-existing vacancies introduced by the EP process itself. These vacancies may trap some fraction of interstitial $\mathrm{H}$ atoms lowering the number of $\mathrm{H}$ atoms available for hydride precipitation upon cooldown leading to smaller hydride sizes translating into higher field onset of strong losses. Same vacancies can trap more $\mathrm{H}$ during mild baking, while in the BCP case all V-H complexes have to be introduced by diffusion.

In summary, we have demonstrated how the nearsurface vacancy structure in niobium is changed by chemical and mild heat treatments and shown how these changes correlate with surface resistance in the superconducting state. The results reveal a strong connection between $\mathrm{V}-\mathrm{H}$ complexes in the magnetic penetration depth and rf dissipation in superconducting niobium cavities. Additionally, our findings suggest that possible presence and effects of $\mathrm{V}-\mathrm{H}$ complexes may be a yet unrecognized contribution in other applications relying on surface superconductivity of niobium.

\section{ACKNOWLEDGMENTS}

The authors would like to acknowledge useful discussions with A. Grassellino and F. Barkov from Fermilab, H. Padamsee from Cornell University, and L. Goncharova from the University of Western Ontario. Fermilab is operated by Fermi Research Alliance, LLC under Contract No. De-AC02-07CH11359 with the United States Department of Energy. The work of A.R. was partly supported by the US DOE Office of Nuclear Physics.

${ }^{1}$ D. C. Mattis and J. Bardeen, Phys. Rev., 111, 412 (1958).

${ }^{2}$ A. A. Abrikosov, L. P. Gor'kov, and I. M. Khalatnikov, Sov. Phys. JETP, 8, 182 (1959).

${ }^{3}$ H. Padamsee, Supercond. Sci. Tech., 14, R28 (2001).

${ }^{4} \mathrm{H}$. Padamsee, RF Superconductivity: Volume II: Science, Technology and Applications (Wiley-VCH Verlag $\mathrm{GmbH}$ and Co., KGaA, Weinheim, 2009).

${ }^{5}$ J. Zmuidzinas, Annu. Rev. Condens. Matter Phys., 3, 169 (2012).

${ }^{6}$ J. M. Raimond, M. Brune, and S. Haroche, Rev. Mod. Phys., 73, 565 (2001).

${ }^{7}$ R. H. Hadfield, Nature Photonics, 3, 696 (2009)

${ }^{8}$ H. Padamsee, J. Knobloch, and T. Hays, RF Superconductivity for Accelerators (John Wiley and Sons, 1998).

${ }^{9}$ J. P. Charrier, B. Coadou, and B. Visentin, in Proceedings of the EPAC'98 (1998) pp. 1885-1887.

${ }^{10} \mathrm{P}$. Kneisel, in Proceedings of the 9th Workshop on RF Superconductivity, TuP044 (Santa Fe, New Mexico, USA, 1999).

${ }^{11}$ G. Eremeev and H. Padamsee, Physica C, 441, 134 (2006).

${ }^{12}$ A. Romanenko, A. Grassellino, F. Barkov, and J. P. Ozelis, Phys. Rev. ST Accel. Beams, 16, 012001 (2013).

${ }^{13} \mathrm{~A}$. Romanenko and A. Grassellino, arXiv preprint arXiv:1304.4516 (2013) 
${ }^{14}$ C. Z. Antoine, "Materials and surface aspects in the development of SRF niobium cavities," EuCARD-BOO-2012-001.

${ }^{15}$ J. Knobloch, R. L. Geng, M. Liepe, and H. Padamsee, in Proceedings of the 9th Workshop on RF Superconductivity, TUA004 (1999).

${ }^{16} \mathrm{C}$. Benvenuti, S. Calatroni, and V. Ruzinov, in Proceedings of the 10th Workshop on RF Superconductivity (Tsukuba, Japan, 2001) p. 441.

${ }^{17}$ M. Delheusy, A. Stierle, N. Kasper, R. P. Kurta, A. Vlad, H. Dosch, C. Antoine, A. Resta, E. Lundgren, and J. Andersen, Appl. Phys. Lett., 92, 101911 (2008).

${ }^{18}$ T. Proslier, J. F. Zasadzinski, L. Cooley, C. Antoine, J. Moore, J. Norem, M. Pellin, and K. E. Gray, Appl. Phys. Lett., 92, 212505 (2008).

${ }^{19}$ A. Romanenko, JRF Materials Workshop (2008).

${ }^{20}$ A. Romanenko and H. Padamsee, Supercond. Sci. Tech., 23, 045008 (2010).

${ }^{21}$ B. Visentin, M. F. Barthe, V. Moineau, and P. Desgardin, Phys. Rev. ST Accel. Beams, 13, 052002 (2010)

${ }^{22}$ A. Romanenko, F. Barkov, L. D. Cooley, and A. Grassellino, Supercond. Sci. Tech., 26, 035003 (2013).

${ }^{23}$ A. Romanenko and L. V. Goncharova, Supercond. Sci. Tech., 24, 105017 (2011).

${ }^{24}$ B. Aune, R. Bandelmann, D. Bloess, B. Bonin, A. Bosotti, M. Champion, C. Crawford, G. Deppe, B. Dwersteg, D. A. Edwards, H. T. Edwards, M. Ferrario, M. Fouaidy, P.-D. Gall, A. Gamp, A. Gössel, J. Graber, D. Hubert, M. Hüning, M. Juillard, T. Junquera, H. Kaiser, G. Kreps, M. Kuchnir, R. Lange, M. Leenen, M. Liepe, L. Lilje, A. Matheisen, W.-D. Möller, A. Mosnier, H. Padamsee, C. Pagani, M. Pekeler, H.-B. Peters, O. Peters, D. Proch, K. Rehlich, D. Reschke, H. Safa, T. Schilcher, P. Schmüser, J. Sekutowicz, S. Simrock, W. Singer,
M. Tigner, D. Trines, K. Twarowski, G. Weichert, J. Weisend, J. Wojtkiewicz, S. Wolff, and K. Zapfe, Phys. Rev. ST Accel. Beams, 3, 092001 (2000).

${ }^{25}$ J. Knobloch, Tech. Rep. SRF 910927-07 (Cornell Univ., 1991).

${ }^{26}$ J. Knobloch, H. Muller, and H. Padamsee, Rev. Sci. Instrum., 65, 3521 (1994).

${ }^{27}$ M. Clement, J. M. M. de Nijs, P. Balk, H. Schut, and A. van Veen, J. Appl. Phys., 79, 9029 (1996).

${ }^{28}$ Y. Fukai and N. Okuma, Phys. Rev. Lett., 73, 1640 (1994).

${ }^{29}$ Y. Fukai, The Metal-Hydrogen System (Springer, BerlinHeidelberg, 2005).

${ }^{30}$ Y. Fukai, J. Alloys Compd., 356-357, 263 (2003).

${ }^{31}$ J. Cizek, I. Prochazka, F. Becvar, R. Kuzel, M. Cieslar, G. Brauer, W. Anwand, R. Kirchheim, and A. Pundt, Phys. Rev. B, 69, 224106 (2004).

32 J. Cizek, I. Prochazka, R. Kuzel, F. Becvar, M. Cieslar, G. Brauer, W. Anwand, R. Kirchheim, and A. Pundt, J. Alloys Compd., 404-406, 580 (2005).

${ }^{33}$ J. Cizek, I. Prochazka, S. Danis, G. Brauer, W. Anwand, R. Gemma, E. Nikitin, R. Kirchheim, A. Pundt, and R. Islamgaliev, Phys. Rev. B, 79, 054108 (2009).

${ }^{34}$ H. Koike, Y. Shizuku, A. Yazaki, and Y. Fukai, J. Phys. Cond. Matt., 16, 1335 (2004).

${ }^{35}$ T. Tajima, R. L. Edwards, F. L. Krawczyk, J. Liu, D. L. Schrage, A. H. Shapiro, J. R. Tesmer, C. J. Wetteland, and R. L. Geng, in Proceedings of the 11th Workshop on RF Superconductivity, THP19 (2003).

${ }^{36}$ K. Saito and P. Kneisel, in Proceedings of the 5th Workshop on RF Superconductivity (DESY, Hamburg, Germany, 1991) pp. 665-679.

${ }^{37}$ A. Grassellino, A. Romanenko, F. Barkov, and A. Suter, $\| \Gamma F-$ SRF'2012. 
(a)

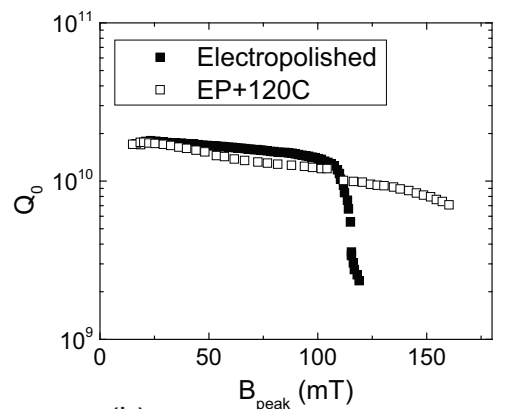

(b)

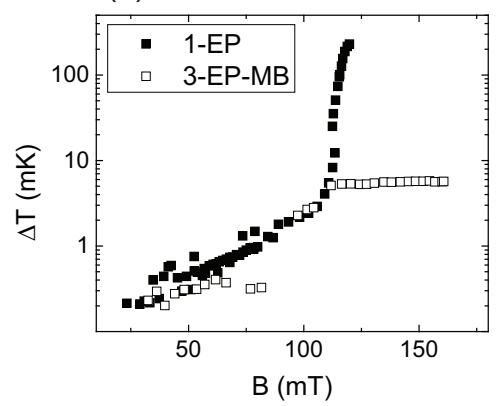

(c)



(d)

(f)

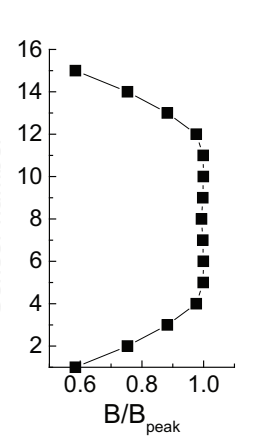



(e)

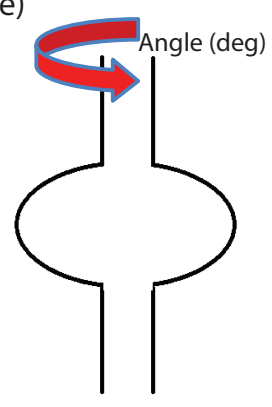





FIG. 1. (a) Results of $Q_{0}\left(B_{\text {peak }}\right)$ measurements on fine grain electropolished cavities before and after mild baking; (b) individual $\Delta T(B)$ for samples 1-EP and 3-EP-MB; (c) temperature mapping boards attached to the outside cavity walls; three boards are removed for demonstration purposes; (d) single individual board with sensor numbering; (e) schematic showing how angle is measured around the rotational cavity axis; 36 boards are uniformly spaced with $10^{\circ}$ separation; (f) local magnetic field $B$ at sensor locations and unfolded temperature maps of $\Delta T \propto R_{\mathrm{S}}$ for unbaked and baked fine grain electropolished cavities at $B_{\text {peak }}=119 \mathrm{mT}$.

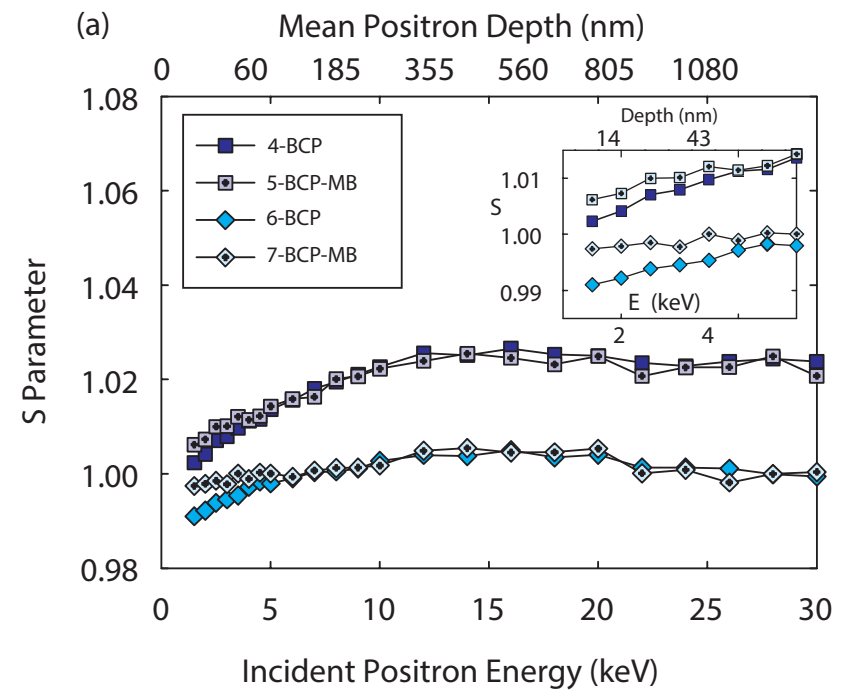

(b)

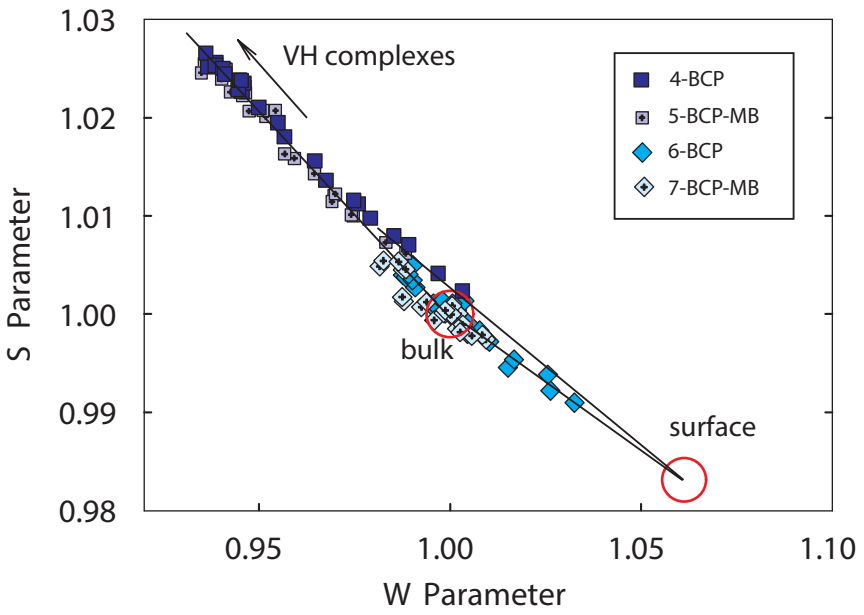

FIG. 2. a) $S(E)$ for samples 6-BCP and 7-BCP-MB from the large grain BCP cavity, and fine grain cavity samples 4-BCP and 5-BCP-MB, which had additional BCP $50 \mu \mathrm{m}$ after they were cut out; b) S-W plot for the same samples. 




FIG. 3. $S(E)$ for fine grain electropolished samples before (1EP, 2-EP), and after (1-EP-MB, 2-EP-MB, 3-EP-MB) mild baking at $120^{\circ} \mathrm{C}$ vacuum bake for 48 hours. Inset shows the change in $S(E)$ shape at low energies.

TABLE I. List of cutout samples investigated with VEPAS.

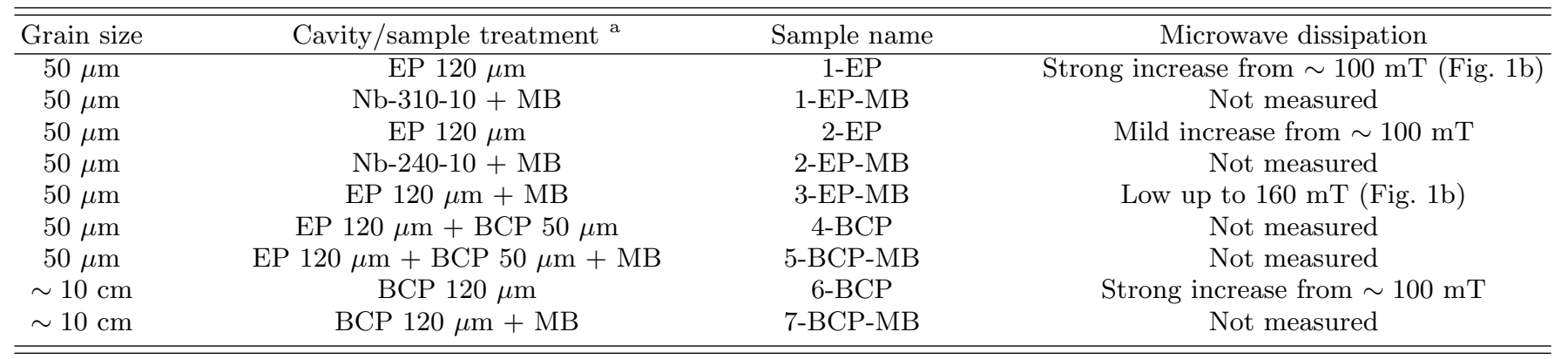

${ }^{\mathrm{a}} \mathrm{EP}=$ electropolishing, $\mathrm{BCP}=$ buffered chemical polishing, $\mathrm{MB}=$ mild baking - vacuum bake at $120^{\circ} \mathrm{C}$ for 48 hours. 